\title{
Noradrenergic Stimulation Enhances Human Action Monitoring
}

\author{
Jordi Riba, ${ }^{1,3}$ Antoni Rodríguez-Fornells, ${ }^{2}$ Adelaida Morte, ${ }^{1}$ Thomas F. Münte, ${ }^{3}$ and Manel J. Barbanoj ${ }^{1}$ \\ ${ }^{1}$ Centre d'Investigació de Medicaments, Institut de Recerca, Servei de Farmacologia Clínica, Hospital de la Santa Creu i Sant Pau, Departament de \\ Farmacologia i Terapèutica, Universitat Autònoma de Barcelona, 08025 Barcelona, Spain, ${ }^{2}$ Faculty of Psychology, University of Barcelona and Institució \\ Catalana de Recerca i Estudis Avançats, 08035 Barcelona, Spain, and ${ }^{3}$ Department of Neuropsychology, Otto von Guericke University, Universitätsplatz 2 , \\ 39106 Magdeburg, Germany
}

Noradrenergic neurotransmission has been associated with the modulation of higher cognitive functions mediated by the prefrontal cortex. In the present study, the impact of noradrenergic stimulation on the human action-monitoring system, as indexed by eventrelated brain potentials, was examined. After the administration of a placebo or the selective $\alpha_{2}$-adrenoceptor antagonist yohimbine, which stimulates firing in the locus ceruleus and noradrenaline release, electroencephalograpic recordings were obtained from healthy volunteers performing a letter flanker task. Yohimbine led to an increase in the amplitude of the error-related negativity in conjunction with a significant reduction of action errors. Reaction times were unchanged, and the drug did not modify the N2 in congruent versus incongruent trials, a measure of preresponse conflict, or posterror adjustments as measured by posterror slowing of reaction time. The present findings suggest that the locus ceruleus-noradrenaline system exerts a rather specific effect on human action monitoring.

Key words: action monitoring; prefrontal cortex; ERN; yohimbine; noradrenaline; human

\section{Introduction}

Noradrenergic neurotransmission has been associated with the regulation of various cognitive processes, including vigilance, attention, working memory, and executive function (Berridge and Waterhouse, 2003). Optimal function of the prefrontal cortex has been postulated to depend on adequate noradrenaline release (Arnsten, 1998). Studies in monkeys have demonstrated the involvement of the locus ceruleus-noradrenaline system in executive tasks (Usher et al., 1999), in which adequate noradrenergic tone leads to improvements of performance in goal-directed behaviors (Aston-Jones et al., 2000). In humans, guanfacine, an adrenergic agonist acting predominantly at postsynaptic receptors at the prefrontal cortex, improves planning and spatial working memory (Jakala et al., 1999). This compound also decreases omission and commission errors in children with attentiondeficit hyperactivity disorder (Scahill et al., 2001), thus hinting at enhanced action monitoring in these patients.

The human action-monitoring system has been the subject of intensive research using both functional neuroimaging measures (Ullsperger and von Cramon, 2001; Kerns et al., 2004) and electrophysiological measures (Gehring et al., 1993; Falkenstein et al., 1995; Vidal et al., 2000; Rodríguez-Fornells et al., 2002). Regarding the latter, the discovery of the error-related negativity (ERN),

Received 0ct. 27, 2004; revised Feb. 25, 2005; accepted March 7, 2005.

This work was supported by Fondo de Investigación Sanitaria Grant 99/0502 from the Spanish Ministry of Health. J.R. is a fellow of the Alexander von Humboldt Foundation. T.F.M. is supported by the Deutsche Forschungsgemeinschaft (MU1311/11-2). A.R.-F. is supported by a Spanish research grant from the Ministerio de Ciencia y Technología. We thank Llúcia Benito, David Martínez, and Sylvie Cotxet for their assistance during data collection.

Correspondence should be addressed to Dr. Jordi Riba at the above address. E-mail: jordi.riba@nat.uni-magdeburg.de. DOl:10.1523/JNEUROSCI.4437-04.2005

Copyright $\odot 2005$ Society for Neuroscience $\quad 0270-6474 / 05 / 254370-05 \$ 15.00 / 0$ a component of the event-related potential that is obtained in response-locked averages with a peak at $\sim 60-100 \mathrm{~ms}$ after an erroneous response, and a frontocentral maximum has been important. The ERN has been interpreted as a correlate of the errordetection process (Holroyd and Coles, 2002) or as a reflection of response conflict arising because of the simultaneous activation of the correct and incorrect response (Cohen et al., 2000; Botvinick et al., 2001). Dipole source modeling (Dehaene et al., 1994; Luu and Tucker, 2001) suggests neural generators in the anterior cingulate cortex (ACC) and/or adjacent structures.

An important theoretical proposal views the ERN in the context of reinforcement learning and attributes the ERN to phasic changes in firing of dopaminergic projections from the mesencephalon to the ACC (Holroyd and Coles, 2002). Specifically, a phasic inhibition of firing is thought to occur when an error is committed leading to a disinhibition of ACC neurons giving rise to the ERN. When the basal ganglia predict that ongoing events are "worse than expected," they produce a negative error signal, whereas predictions that ongoing events are "better than expected" lead to a positive error signal (Barto, 1995; Houk et al., 1995; Montague et al., 1996). It is these negative and positive error signals that lead to the phasic decreases and increases in the tonic activity of the mesencephalic dopamine system. The dopamine signals to the ACC are used to optimize its filtering function.

In addition to dopaminergic input, noradrenergic fibers stemming from the locus ceruleus also project to the ACC, providing an alternative and/or complementary source of modulation for this region (Berger, 1992). In the present study, we evaluated the effects of the selective $\alpha_{2}$-adrenoceptor antagonist yohimbine, a drug that increases firing in the locus ceruleus (Ivanov and 
Table 1. Behavioral measures in each treatment condition expressed as mean (SD)

\begin{tabular}{|c|c|c|c|c|}
\hline & \multicolumn{4}{|c|}{ Reaction time and performance data } \\
\hline & \multicolumn{2}{|l|}{ Placebo } & \multicolumn{2}{|l|}{ Yohimbine } \\
\hline & Congruent & Incongruent & Congruent & Incongruent \\
\hline \multicolumn{5}{|l|}{ Compatibility } \\
\hline RT correct & $316(34)$ & $338(34)$ & $314(28)$ & $340(26)$ \\
\hline RT errors & $263(28)$ & $274(27)$ & $266(21)$ & $275(15)$ \\
\hline Percentage of errors & $13.1(4.7)$ & $26.8(9.4)$ & $10.6(5.2)$ & $23.7(7.9)$ \\
\hline Percentage of corrections & $92.7(7.8)$ & $94.4(5.8)$ & $93.7(6.9)$ & $95.05(4.2)$ \\
\hline \multicolumn{5}{|l|}{ Posterror adjustments } \\
\hline RT after correct & $325(39)$ & & $323(27)$ & \\
\hline RT after error & $340(41)$ & & $344(38)$ & \\
\hline$n$ errors after errors & $42(30)$ & & $32(28)$ & \\
\hline
\end{tabular}

RTs are expressed in milliseconds.

Aston-Jones, 1995) and noradrenaline release at the synapse (Starke et al., 1989) by blocking the autoreceptor-regulated negative-feedback loop (Langer, 1997).

\section{Materials and Methods}

Subjects. Fifteen right-handed volunteers (seven men, eight women) with a mean of 24.8 years of age (range, 20-37) participated in the study. Medical history, laboratory tests, electrocardiogram, and urinalysis were normal, and no medication or illicit drugs were allowed 2 weeks before the study. Subjects also abstained from alcohol, tobacco, and caffeinated drinks for $48 \mathrm{~h}$ before each experimental day. The study was conducted in accordance with the Declarations of Helsinki and Tokyo and was approved by the Hospital Ethics Committee and the Spanish Ministry of Health.

Study design. Oral doses of $30 \mathrm{mg}$ of yohimbine and placebo (lactose) packaged in identical capsules were administered in a balanced order according to a double-blind randomized within-subjects design. The two experimental days were separated by at least 1 week washout period. After arrival in the laboratory under fasting conditions, a urine sample was obtained to test for illicit drug intake, electrodes were applied to the scalp, and medication was given. During each recording session, volunteers remained in a quiet room and were asked to stay alert throughout the experiment. The current experiment was conducted $2 \mathrm{~h}$ after drug administration.

Stimuli and procedure. The Eriksen flanker task (Eriksen and Eriksen, 1974) was used, which requires a response to the center letter of a fiveletter array with either a left-hand (letter $\mathrm{H}$ ) or right-hand (letter S) response. Additional letters flanking the target letter either favored the target response (congruent trials, HHHHH or SSSSS) or primed the other response (incongruent trials, HHSHH or SSHSS). To increase the number of errors produced, $60 \%$ of the trials were incongruent. Each stimulus array subtended $\sim 2.5^{\circ}$ of visual angle in width, and a fixation cross was presented in the middle of the computer monitor just below the target letter in the array. The duration of the stimuli was $100 \mathrm{~ms}$, and a random stimulus onset asynchrony between 900 and $1100 \mathrm{~ms}$ was used. Letter/hand assignments were counterbalanced between subjects and maintained in both sessions. Before the first experimental session, subjects were trained with 200 trials to reach a reaction time (RT) baseline level, and they were given feedback about their performance. The goal of this procedure was to aim for a reaction time that would yield $\sim 10-15 \%$ of errors. The experiment proper consisted of six blocks of $4 \mathrm{~min}$ and 200 stimuli each. A 30 s rest period was allowed between blocks. Subjects were required to respond to the stimuli as fast as possible and to correct their errors as fast as possible whenever they detected them.

Electrophysiological recording. The electroencephalogram was recorded from the scalp using gold electrodes located at 29 standard positions (Fp1/2, F3/4, C3/4, T3/4, T5/6, P3/4, O1/2, F7/8, Fz, Cz, Pz, Fc3/4, $\mathrm{FT} 7 / 8, \mathrm{Cp} 3 / 4, \mathrm{TP} 7 / 8, \mathrm{PO} 3 / 4)$. Biosignals were rereferenced off-line to the mean of the activity at the two mastoid leads. Vertical eye movements were monitored with an electrode at the infraorbital ridge of the left eye. Electrode impedances were kept $<5 \mathrm{k} \Omega$. The electrophysiological signals were filtered with a bandpass of $0.1-50 \mathrm{~Hz}$ and digitized at a rate of $250 \mathrm{~Hz}$. Trials in which base-to-peak electrooculogram amplitude was $>100 \mu \mathrm{V}$, amplifier saturation occurred, or the baseline shift was $>300 \mu \mathrm{V} / \mathrm{s}$ were automatically rejected.

Response-locked event-related brain potentials (ERPs) were averaged starting $400 \mathrm{~ms}$ before the response of the subject until $624 \mathrm{~ms}$ after response onset. The baseline used for the response-locked ERN was between -150 and $-50 \mathrm{~ms}$. Stimulus-locked ERPs were averaged over epochs of $1024 \mathrm{~ms}$ starting $100 \mathrm{~ms}$ before the stimulus. The mean amplitude value in the interval between -100 and $0 \mathrm{~ms}$ was used as baseline. Two types of trials were averaged separately: correct responses and errors. The resulting ERPs were filtered with a low-pass filter (12 Hz half-amplitude cutoff).

ERP effects were quantified for the three midline electrodes $(\mathrm{Fz}, \mathrm{Cz}$, and $\mathrm{Pz}$ ), and the resulting data were subjected to repeated-measures ANOVAs with the Greenhouse-Geisser $\epsilon$ correction applied when necessary. The original degrees of freedom and corrected $p$ values are given in Results.

\section{Results}

\section{Behavioral analysis}

Main behavioral results are shown in Table 1. A main effect of the type of response reflected faster reaction times for erroneous responses (correct, $327 \pm 33 \mathrm{~ms}$; erroneous, $269 \pm 23 \mathrm{~ms} ; F_{(1,14)}=$ $131 ; p<0.001)$. Congruent trials were faster than incongruent trials $\left(F_{(1,14)}=77 ; p<0.001\right)$, although this effect was larger in correct than in incorrect trials (type of trial $\times$ compatibility, $\left.F_{(1,14)}=35 ; p<0.001\right)$. Neither a main effect of treatment $(F<$ 1) nor treatment by type of response or treatment by compatibility (both $F<1$ ) were found. The time needed to correct an erroneous response did not differ regarding compatibility or treatment $(F<1$, in both cases; mean corrective reaction time: placebo, $191 \pm 53 \mathrm{~ms}$; yohimbine, $186 \pm 49 \mathrm{~ms}$ ).

The same analysis was performed on the percentage of erroneous responses (corrected and uncorrected). This value was higher in the incongruent condition compared with the congruent condition $\left(F_{(1,14)}=56.9 ; p<0.001\right)$ (Table 1$)$. In the yohimbine condition, subjects committed less erroneous responses $\left(F_{(1,14)}=5.45 ; p<0.05\right)$. The interaction between compatibility and treatment was not significant $(F<1)$. Regarding the percentage of erroneous responses that were afterward corrected, no significant differences were found for treatment $(F<1)$, compatibility $\left(F_{(1,14)}=3.3 ; p>0.09\right)$, or their interaction $(F<1)$.

Posterror adjustments were measured by assessing posterror slowing and the number of errors after erroneous responses. Reaction times to correct trials immediately following a correct or an erroneous response, respectively, are shown in Table 1. A twoway ANOVA showed a main effect of type of preceding response (correct vs erroneous; $F_{(1,14)}=12.09 ; p<0.01$ ) but no effect of treatment $(F<1)$ and no preceding response by treatment interaction $\left(F_{(1,14)}=2.04 ; p<0.2\right)$. On the contrary, a marginally significant decrease was observed in the number of errors that followed an erroneous response (Table 1) after yohimbine administration $\left(F_{(1,14)}=4.4 ; p=0.054\right)$.

\section{ERP analysis}

The percentage of EEG epochs rejected as a result of artifacts did not differ between treatments $\left(F_{(1,14)}<1\right.$; placebo, $14 \%$; yohimbine, $16 \%)$. The mean number of errors entered into the average 
after artifact rejection was $227 \pm 72$ for placebo and $207 \pm 72$ for yohimbine $\left(F_{(1,14)}=2.75 ; p>0.10\right)$.

The response-locked averages are depicted in Figure $1 A$. Although no medication differences are apparent for the correct trials, the ERN component in the error trials was increased in the yohimbine condition. A mean amplitude measure in the time window of $0-100 \mathrm{~ms}(\mathrm{Fz}, \mathrm{Cz}$, and $\mathrm{Pz})$ yielded a main effect of type of response $\left(F_{(1,14)}=71.1 ; p<0.001\right)$. Although there was no main effect of treatment $\left(F_{(1,14)}=\right.$ $3.7 ; p>0.07)$, a significant interaction between type of response and treatment $\left(F_{(1,14)}=8.27 ; p<0.012\right)$ reflected the selective effect of yohimbine on erroneous responses.

Post hoc analyses on the error trials revealed that yohimbine increased the ERN component for both $\mathrm{Fz}\left(F_{(1,14)}=4.6\right.$; $p<$ $0.05)$ and $\mathrm{Cz}\left(F_{(1,14)}=11.2 ; p<0.005\right)$ electrode sites.

To check whether the reduced error rate observed after yohimbine accounted for the amplitude increases in the ERN, the difference in error rate between the yohimbine condition and placebo was introduced as a covariate in the ANOVA. This analysis yielded again a significant type of response by treatment interaction at $\mathrm{Cz}$ $\left(F_{(1,13)}=7.1 ; p<0.05\right)$, indicating that the drug-induced enhancement of the ERN persisted after controlling for differences in performance accuracy.

A typical frontocentral distribution was found for the ERN (Fig. 1C), and the subtraction (yohimbine minus placebo, error trials) showed a similar distribution, indicating that the effect of yohimbine was on the ERN component.

To test for treatment effects on the error positivity $(\mathrm{Pe})$ (i.e., the positive component that follows the ERN) (Fig. $1 A$ ), we computed the peak amplitude for this component at $\mathrm{Pz}$ in the erroneous trials (time window, 100-400 ms). No significant differences were found between placebo and yohimbine either in amplitude $(F<1$; placebo, $5.4 \pm 2.7 \mu \mathrm{V}$; yohimbine, $5.1 \pm 2.7 \mu \mathrm{V})$ or in latency for this component $(F<1$; placebo, $190 \pm 66 \mathrm{~ms}$; yohimbine, $189 \pm$ $56 \mathrm{~ms})$.

\section{Stimulus-locked ERPs}

The stimulus-locked averages (Fig. $1 B$ ) showed no major differences resulting from treatment for the early ERP components (N1 or P2). At $\sim 250 \mathrm{~ms}$, a negative component (N2) was observed, which was somewhat broader in the erroneous trials. The $\mathrm{N} 2$ was quantified by a mean amplitude measure (250-450 ms). Errors showed an increased negativity when compared with correct responses (type of response, $F_{(1,14)}=28.8 ; p<0.001$ ). Treatment $\left(F_{(1,14)}=1.8 ; p>0.13\right)$ and the interaction between type of response and treatment were not significant $\left(F_{(1,14)}=2.9 ; p>\right.$ $0.10)$. However, a significant interaction between treatment and type of response and electrode was found $\left(F_{(2,28)}=5.18 ; p<\right.$
B.

\section{Correct Error}

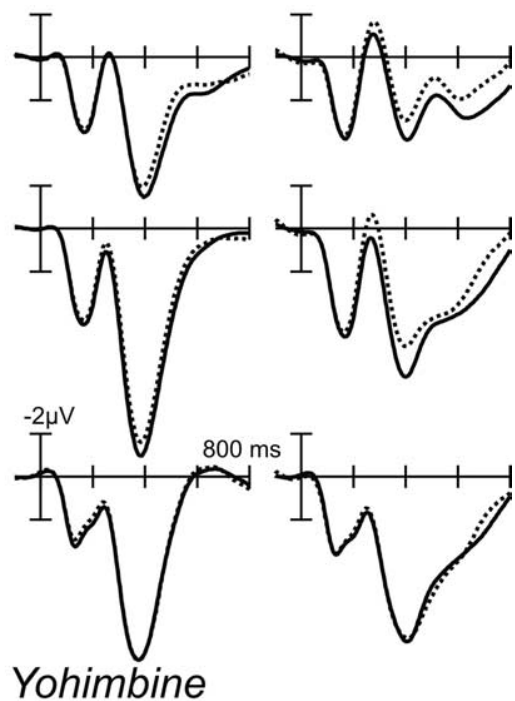

Yohimbine

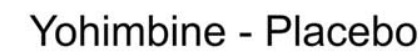

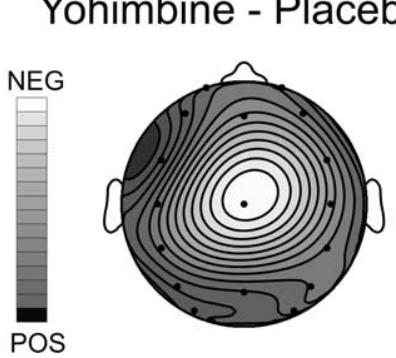

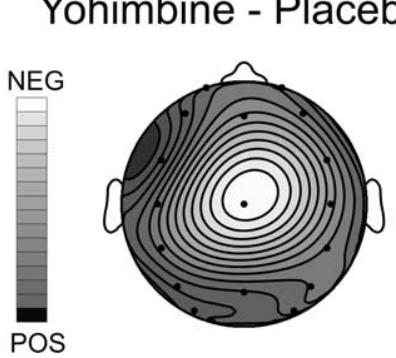

Figure 1. A, Response-locked ERPs depicted for correct and erroneous responses in each treatment condition (placebo and

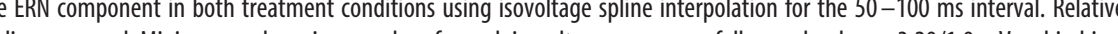
scaling was used. Minimum and maximum values for each isovoltage map are as follows: placebo, $-3.29 / 1.9 \mu \mathrm{V}$; yohimbine, $-4.9 / 1.68 \mu \mathrm{V}$; yohimbine minus placebo, $-1.69 / 0.08 \mu \mathrm{V}$.

0.012). Pairwise comparisons of the error trials revealed that yohimbine increased negativity mainly at $\mathrm{Cz}\left(F_{(1,14)}=6.8 ; p<\right.$ 0.020).

Previous studies have found a larger N2 in incongruent compared with congruent trials (Van Veen and Carter, 2002). This was also found in the present study (Fig. 2). An ANOVA was performed on a mean amplitude measure (250-350 ms time window) for correct trials at midline locations, with compatibility and treatment as within-subject factors. As expected, incongruent trials (Fig. 2A) showed an increase in the frontal N2 component compared with congruent trials. This effect was significant $\left(F_{(1,14)}=28.9 ; p<0.001\right.$; congruent, $4.3 \pm 4.3 \mu \mathrm{V}$; incongruent, $2.5 \pm 4.2 \mu \mathrm{V})$. Neither treatment $\left(F_{(1,14)}<1\right)$ nor the interaction between treatment and compatibility $\left(F_{(1,14)}<1\right)$ showed any significant effect.

To confirm the lack of an effect of yohimbine on the N2 amplitude difference in incongruent versus congruent correct trials, the incongruent-congruent difference waves were obtained (Fig. $2 B$ ), and the peak values in the 250-450 ms time range were submitted to statistical analysis. Again, repeated-measures 
A

Placebo

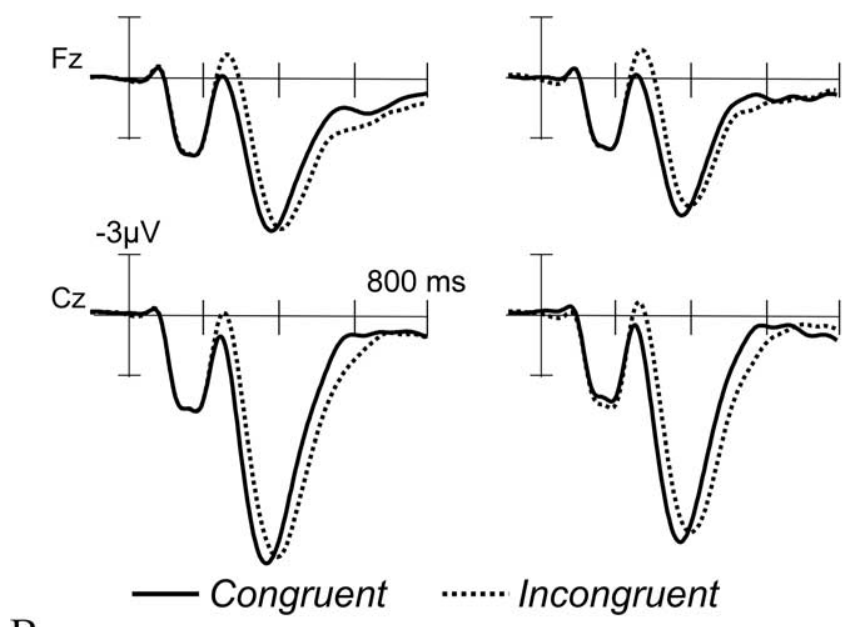

B

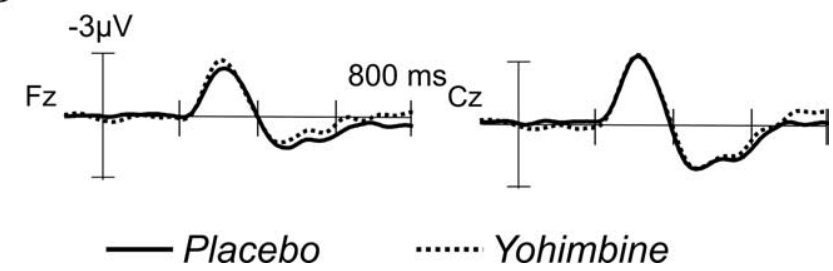

Figure 2. A, Stimulus-locked ERPs for congruent and incongruent trials at $\mathrm{Fz}$ and $\mathrm{CZ}$, showing an increase in the amplitude of the $\mathrm{N} 2$ component in the incongruent trials. $\boldsymbol{B}$, Incongruent minus congruent difference waves at $\mathrm{Fz}$ and $\mathrm{C} z$ for the placebo and yohimbine conditions. The congruency effect on the $\mathrm{N} 2$ was not modified by yohimbine administration.

ANOVAs with treatment as a factor did not show any significant peak amplitude differences either at $\mathrm{Fz},\left(F_{(1,14)}=1.6 ; p=0.2\right)$ or at $\mathrm{Cz}\left(F_{(1,14)}<1\right)$.

The stimulus-locked averages suggest that there is no medication effect on the P3 component. Indeed, a peak amplitude measure (time window, 200-600 ms; Pz electrode) revealed no main effect of type of response or treatment. Also, the interaction of type of response and treatment was nonsignificant $($ all $F<1)$. A P3 peak latency measure (same time window) demonstrated a delay for erroneous trials $\left(F_{(1,14)}=41.3 ; p<0.001\right.$; correct responses, $372 \pm 38 \mathrm{~ms}$; erroneous, $425 \pm 64 \mathrm{~ms})$. There were no treatment effects on P3 latency, however, and the interaction between treatment and type of response was nonsignificant (both $F<1$ ).

\section{Discussion}

Noradrenergic stimulation appears to selectively enhance electrophysiological correlates of action monitoring in humans. The selective $\alpha_{2}$-adrenoceptor antagonist yohimbine decreased the number of action errors in the Eriksen flanker task and increased the amplitude of the ERN. No effects were observed on reaction times, posterror slowing, or other components of the ERP. Specifically, the amplitude difference of the N2 component of the stimulus-locked ERPs in the congruent versus incongruent correct responses, which has been interpreted as an indicator of response conflict, was not modified by yohimbine. These findings are the first to demonstrate the facilitatory effects of increased noradrenergic activity on the human error-monitoring system. Previous studies with different paradigms did not reveal robust effects of yohimbine on ERPs. For example, in a choice reaction time task, it modified neither the reaction times nor the latencies and amplitudes of the N200 and the P3 components of the visual ERP (Halliday et al., 1994). In another study involving a three-tone target detection task, yohimbine did not modify the latency of the N250 or the P3b associated with target identification and reaction times (Turetsky and Fein, 2002). However, a trend toward an increase in performance accuracy was observed. Interestingly, the drug did modify the orienting response to novel or rare stimuli, speeding $\mathrm{P} 3$ a peak latency and increasing its amplitude. Thus, yohimbine appeared to modify activity of the frontal attentional network, which, like the error-monitoring system, is thought to be subserved by the ACC (Posner and Petersen, 1990).

Previously, drug effects on the ERN as an indicator of the human action-monitoring system have been interpreted in the light of the reinforcement-learning theory (Holroyd and Coles, 2002), which attributes the ERN to phasic changes in firing of the mesencephalon-ACC dopaminergic pathways. Pharmacological investigations have in fact demonstrated a modulatory role of the dopamine system in the generation of the ERN. De Bruijn et al. (2004) observed an enlargement of the ERN and no effect on reaction time or error rates after administration of $15 \mathrm{mg}$ of amphetamine, an indirect dopamine agonist, whereas the dopaminergic antagonist haloperidol ( $3 \mathrm{mg}$; single dose) has been found to attenuate the ERN (Zirnheld et al., 2004). Finally, caffeine ( 3 or $5 \mathrm{mg} / \mathrm{kg}$ body weight), an adenosine receptor antagonist influencing dopaminergic neurotransmission, yielded larger ERNs together with reductions in reaction times and error rates (Tieges et al., 2004).

The present findings show that in addition to the dopamine system, the noradrenergic system might serve as a complementary source of modulation of the ERN. Noradrenergic neurotransmission does not seem to be involved in reward. Rather, the locus ceruleus-noradrenaline system appears to modulate information processing by increasing the signal-to-noise ratio of relevant information (Servan-Schreiber et al., 1990). The activity of this system seems to depend on the salience of the stimulus and to be independent of its emotional valence (for review, see Berridge and Waterhouse, 2003). Thus, even if changes in noradrenergic firing do not constitute the central event generating the ERN, it is plausible that increased noradrenergic neurotransmission leads to a facilitation of activity in the ACC. It is worth mentioning that in the present study, we observed a dissociation of yohimbine effects on the N2 congruency effect and on the ERN. A possible explanation is that the locus ceruleus-noradrenergic system is differently engaged in each situation, in line with a proposed synchronization of activity in the ACC mediated by phasic discharges of the locus ceruleus (Paus, 2001). Additionally, there exists evidence from neuroimaging studies indicating that (pre-) response conflict and error processing may be mediated by different anatomical substrates within the frontomedial cortex (Ullsperger and von Cramon, 2001).

An alternative explanation to the effects of yohimbine on the ERN is that increased noradrenergic tone could enhance the activity of other neural systems in addition to the ACC, thus facilitating stimulus detection and stimulus-response mapping or increasing salience of the error. Although our current results are rather selective for the ERN, our data do not allow us to attribute the effects of yohimbine exclusively to specific increases in activation of the ACC. Other brain areas, such as the lateral prefrontal cortex, interact with the ACC to modulate the ERN (Gehring and Knight, 2000). Activation at this level could have played a role in the present findings. Additionally, it is possible that yohimbine-induced increases in selective attention could have led to the observed enhancement of the ERN. Other studies have 
shown that decreasing attention has a negative impact on ERN amplitude (Pailing and Segalowitz, 2004).

In conclusion, the stimulation of noradrenergic neurotransmission enhanced the ERN and improved performance accuracy in the absence of additional effects on other ERP components or reaction times. The present findings suggest that the locus ceruleus-noradrenaline system exerts a modulatory activity on action monitoring in humans. Future research should identify the subprocesses and neural substrates targeted by, and assess the interplay between, dopaminergic and noradrenergic neurotransmission in the genesis and modulation of the ERN.

\section{References}

Arnsten AFT (1998) Catecholamine modulation of prefrontal cortical cognitive function. Trends Cogn Sci 2:436-447.

Aston-Jones G, Rajkowski J, Cohen J (2000) Locus coeruleus and regulation of behavioral flexibility and attention. Prog Brain Res 126:165-182.

Barto AG (1995) Adaptive critics and the basal ganglia. In: Models of information processing in the basal ganglia (Houk J, Davis J, Beiser D, eds), pp 215-232. Cambridge, MA: MIT.

Berger B (1992) Comparative neurochemical analysis of the frontal cortex, with special emphasis on the dopamine innervation of the primary motor cortex, lateral prefrontal cortex and the anterior cingulate cortex. In: Advances in neurology, Vol 57 (Chauvel P, Delgado-Escueta AV, eds), pp 525-544. New York: Raven.

Berridge CW, Waterhouse BD (2003) The locus coeruleus-noradrenergic system: modulation of behavioral state and state-dependent cognitive processes. Brain Res Brain Res Rev 42:33-84.

Botvinick MM, Braver TS, Barch DM, Carter CS, Cohen JD (2001) Conflict monitoring and cognitive control. Psychol Rev 108:624-652.

Cohen JD, Botvinick M, Carter CS (2000) Anterior cingulate and prefrontal cortex: who's in control? Nat Neurosci 3:421-423.

De Bruijn ERA, Hulstijn W, Verkes RJ, Ruigt GSF, Sabbe BGC (2004) Druginduced stimulation and suppression of action monitoring in healthy volunteers. Psychopharmacology 177:151-160.

Dehaene S, Posner MI, Tucker DM (1994) Localization of a neural system for error detection and compensation. Psychol Sci 5:303-305.

Eriksen BA, Eriksen CW (1974) Effects of noise letters upon the identification of target letters in a non-search task. Percept Psychophys 16:143-149.

Falkenstein M, Hohnsbein J, Hoormann J (1995) Event-related potential correlates of errors in reaction tasks. Electroencephalogr Clin Neurophysiol Suppl 44:287-296.

Gehring WJ, Knight RT (2000) Prefrontal-cingulate interactions in action monitoring. Nat Neurosci 3:516-520.

Gehring WJ, Gross B, Coles MGH, Meyer DE, Donchin E (1993) A neural system for error detection and compensation. Psychol Sci 4:385-390.

Halliday R, Naylor H, Brandeis D, Callaway E, Yano L, Herzig K (1994) The effect of D-amphetamine, clonidine, and yohimbine on human information processing. Psychophysiology 31:331-337.

Holroyd CB, Coles MG (2002) The neural basis of human error processing: reinforcement learning, dopamine, and the error-related negativity. Psychol Rev 109:679-709.

Houk JC, Adams JL, Barto AG (1995) A model of how the basal ganglia generate and use neural signals that predict reinforcement. In: Models of information processing in the basal ganglia (Houk J, Davis J, Beiser D, eds), pp 249-270. Cambridge, MA: MIT.

Ivanov A, Aston-Jones G (1995) Extranuclear dendrites of locus coeruleus neurons: activation by glutamate and modulation of activity by alpha adrenoceptors. J Neurophysiol 74:2427-2436.

Jakala P, Riekkinen M, Sirvio J, Koivisto E, Kejonen K, Vanhanen M, Riekkinen P (1999) Guanfacine, but not clonidine, improves planning and working memory performance in humans. Neuropsychopharmacology 20:460-470.

Kerns JG, Cohen JD, MacDonald III AW, Cho RY, Stenger VA, Carter CS (2004) Anterior cingulate conflict monitoring and adjustments in control. Science 303:1023-1026.

Langer SZ (1997) 25 years since the discovery of presynaptic receptors: present knowledge and future perspectives. Trends Pharmacol Sci 18:95-99.

Luu P, Tucker DM (2001) Regulating action: alternating activation of midline frontal and motor cortical networks. Clin Neurophysiol 112:1295-1306.

Montague PR, Dayan P, Sejnowski TJ (1996) A framework for mesencephalic dopamine systems based on predictive Hebbian learning. J Neurosci 16:1936-1947.

Pailing PE, Segalowitz SJ (2004) The effects of uncertainty in error monitoring on associated ERPs. Brain Cogn 56:215-233.

Paus T (2001) Primate anterior cingulate cortex: where motor control, drive and cognition interface. Nat Rev Neurosci 2:417-424.

Posner MI, Petersen SE (1990) The attention system of the human brain. Annu Rev Neurosci 13:25-42.

Rodríguez-Fornells A, Kurzbuch AR, Münte TF (2002) Time course of error detection and correction in humans: neurophysiological evidence. J Neurosci 22:9990-9996.

Scahill L, Chappell PB, Kim YS, Schultz RT, Katsovich L, Shepherd E, Arnsten AF, Cohen DJ, Leckman JF (2001) A placebo-controlled study of guanfacine in the treatment of children with tic disorders and attention deficit hyperactivity disorder. Am J Psychiatry 158:1067-1074.

Servan-Schreiber D, Printz H, Cohen JD (1990) A network model of catecholamine effects: gain, signal-to-noise ratio, and behavior. Science 249:892-895.

Starke K, Gothert M, Kilbinger H (1989) Modulation of neurotransmitter release by presynaptic autoreceptors. Physiol Rev 69:864-989.

Tieges Z, Richard Ridderinkhof K, Snel J, Kok A (2004) Caffeine strengthens action monitoring: evidence from the error-related negativity. Brain Res Cogn Brain Res 21:87-93.

Turetsky BI, Fein G (2002) $\alpha_{2}$-Noradrenergic effects on ERP and behavioral indices of auditory information processing. Psychophysiology 39:147-157.

Ullsperger M, von Cramon DY (2001) Subprocesses of performance monitoring: a dissociation of error processing and response competition revealed by event-related fMRI and ERPs. NeuroImage 14:1387-1401.

Usher M, Cohen JD, Servan-Schreiber D, Rajkowski J, Aston-Jones G (1999) The role of locus coeruleus in the regulation of cognitive performance. Science 283:549-554.

Van Veen V, Carter CS (2002) The timing of action-monitoring processes in the anterior cingulate cortex. J Cogn Neurosci 14:593-602.

Vidal F, Hasbroucq T, Grapperon J, Bonnet M (2000) Is the "error negativity" specific to errors? Biol Psychol 51:109-128.

Zirnheld PJ, Carroll CA, Kieffaber PD, O'Donnell BF, Shekhar A, Hetrick WP (2004) Haloperidol impairs learning and error-related negativity in humans. J Cogn Neurosci 16:1098-1112. 